\title{
A Metal-free Multicomponent Strategy for Amidine Synthesis
}

\author{
Zayed Alassad $^{1 \ddagger}$, Anas Abo Raed ${ }^{1 \ddagger}$, Meital Shema Mizrachi ${ }^{1}$, Mónica Pérez-Temprano ${ }^{2}$ and Anat \\ Milo $^{1 *}$
}

1Department of Chemistry, Ben-Gurion University of the Negev, Beer Sheva, Israel.

2Institute of Chemical Research of Catalonia (ICIQ), Barcelona Institute of Science and Technology (BIST), Tarragona, Spain.

KEYWORDS. Amidine, Diversity-Oriented Synthesis (DOS), Multi-Component Reactions (MCR), Mechanism, Multivariate Analysis, Late-Stage Functionalization

\begin{abstract}
Amidines are a ubiquitous class of bioactive compounds found in a wide variety of natural products; thus, efficient strategies for their preparation are in great demand. Specifically, their common structural core decorated with three substituents, set amidines as perfect candidates for multicomponent synthesis. Herein, we present a highly modular metalfree multicomponent strategy for the synthesis of sulfonyl amidines. This work was focused on selecting readily accessible reagents to facilitate the in situ formation of enamines by the addition of amines to ketones. These components were coupled with azides to provide a broad reaction scope with respect to all three coupling partners. Aromatic and aliphatic amines and ketones were tolerated under our reaction conditions. Likewise, the presence of a methyl group on the ketone was critical to reactivity, which was leveraged for the design of a highly regioselective reaction with aliphatic ketones. A biologically active compound was successfully synthesized in one step, demonstrating the practical utility of our methodology. Finally, the postulated mechanism was investigated and supported both experimentally and by means of a multivariate statistical model.
\end{abstract}

\section{Introduction}

Multicomponent reactions are arguably one of the most powerful methods for diversity-oriented synthesis..$^{1-5}$ These step-economical strategies have been embraced by medicinal chemists to expand the chemical space evaluated in biological screening and drug discovery, and are predicated on constructing a common structural core from easily diversified building blocks.6,7 These reactions commonly proceed via a cascade of elementary chemical steps. Nevertheless, orthogonal functionality and undesirable cross-reactivity between reaction components are key limiting factors when designing appropriate synthons and conditions. Amidines are a prominent class of bioactive compounds found in a wide variety of natural products (scheme 1.a)..$^{8-10}$ Due to their common structural core decorated with three substituents, amidines are perfect candidates for multicomponent synthesis. ${ }^{11}$ Their unique structural features, stemming from a conjugated amine core, are at the root of numerous applications. ${ }^{10,11}$ In addition to their broad use as bioactive pharmacophores, amidines are synthetic intermediates for heterocyclic compounds and useful ligands for metal complexes. ${ }^{11}$ Nevertheless, none of the existing synthetic methodologies afford amidines with three aromatic substituents. Thus, the design of a multicomponent, single-step reaction to afford a diverse array of amidines, especially those with three aromatic substituents decorating the conjugated amine core, is both synthetically challenging and appealing as a gateway to numerous untapped applications.

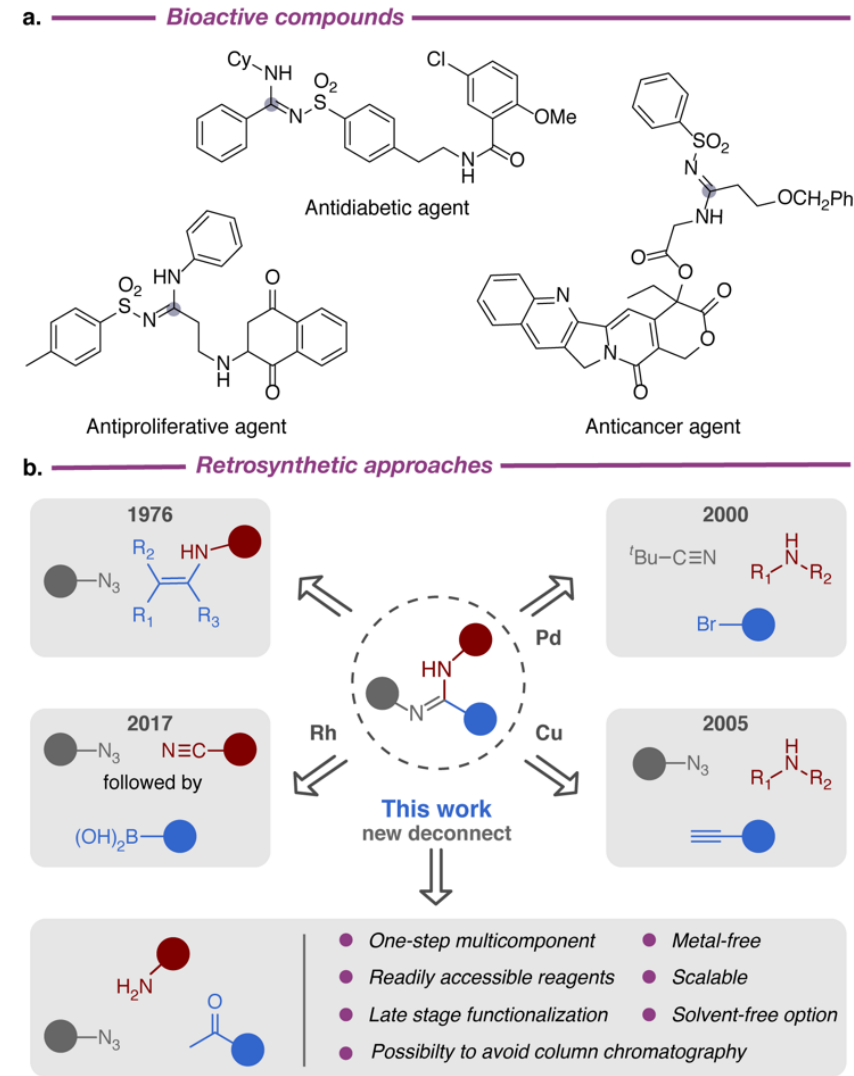

Scheme 1. (a) examples of bioactive compounds bearing an amidine core (b) selected approaches for the preparation of amidines and the methodology presented in this work 
In light of the above-mentioned considerations, it is not surprising that amidine synthesis has received attention in recent years. Initial studies were based on the reactivity between enamines and sulfonyl azides; however, this methodology depended on the isolation of stable enamine substrates (Scheme 1.b. top left). ${ }^{12-14}$ In the following years, focus shifted to organometallic catalysis. Furber and coworkers developed a palladium-catalyzed multicomponent amidine synthesis from secondary amines, tert-butyl isonitrile, and substituted bromobenzene (Scheme 1.b. top right). ${ }^{15}$ However, this transformation required the addition of stoichiometric amounts of base $\left(\mathrm{Cs}_{2} \mathrm{CO}_{3}\right)$, and the substrate scope was limited mostly to aliphatic secondary amines and only one example of isonitrile precursor was presented. Chang and coworkers reported a copper-catalyzed multicomponent oxidative amination, which offers practical access to sulfonyl amidines from alkyl amine, azide and alkyne building blocks (Scheme 1.b. bottom right). ${ }^{16}$ Zhang and coworkers reported a one-pot two-step rhodium-catalyzed amidine synthesis, wherein isonitriles were reacted with azides forming carbodiimides, to which boronic acids were added to afford amidine products (Scheme 1.b. bottom left). ${ }^{17}$ The scope of this reaction was limited to tert-butyl, cyclohexyl and 2,6-dimethylphenyl isonitrile, and only allylic and aromatic boronic acids were used. Overall, despite the great progress these and other metal-catalyzed methodologies represent in the multicomponent synthesis of amidines, they still rely on the preparation of building blocks that are often not readily accessible such as isonitriles, aldoximes, and terminal alkynes. ${ }^{18,19}$

Table 1. Perturbations to optimal reaction conditions

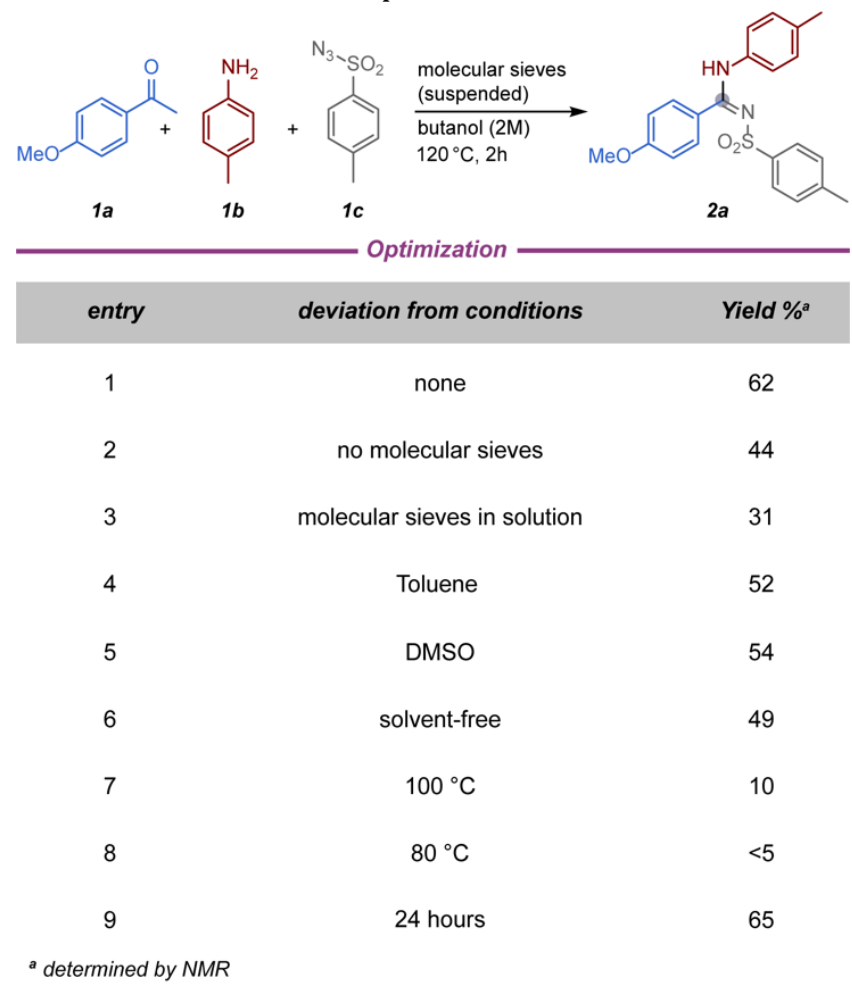

Inspired by the metal-free approach for the formation of sulfonyl amidines through a reaction between enamines and azides (Scheme 1.b.), ${ }^{12,20}$ we envisioned a new strategy for amidine synthesis. Herein, based on the in situ formation of enamines by the addition of amines to ketones, we disclose a three-component, one-step, metal-free strategy to afford sulfonyl amidines from readily accessible reagents. To the best of our knowledge, this is the first transformation in which three aromatic substituents could be connected to the amidine core in a single step.

\section{Methodology Optimization and Scope}

To develop and optimize this reaction, three model components that can be tracked by NMR were selected: acetophenone $\mathbf{1 a}$, aniline $\mathbf{1 b}$, and azide 1c. Our starting point was to apply conditions that resemble the aforementioned initial studies; ${ }^{21,22}$ thus, alcohols seemed to be an ideal solvent choice. Because conditions that are known to facilitate imine formation between acetophenone and aniline, such as elevated reaction temperatures, were also required, butanol was selected due to its high boiling point. Thus, we placed all three components under reflux in butanol and to our delight $44 \%$ of the desired product was obtained (Table1. entry 2). Based on the hypothesized reaction mechanism, we speculated that molecular sieves (MS) could have a positive effect on the conversion by sequestering water released in the formation of the imine intermediate. However, upon the addition of MS to the reaction mixture the yield decreased (Table1. entry 3 ). We suspected that the basicity of molecular sieves could have negatively affected the conversion. Thus, we suspended MS at the top of the reaction tube with filter paper (see Figure S1 in SI), which led to a significant increase in reaction yield (Table1. entry 1). We then tested toluene and DMSO as solvents because they are commonly used in imine formation reactions, ${ }^{23,24}$ yet in both cases the yield was slightly diminished (Table1. entry 4, 5). We also tested a solvent-free reaction and maintained decent yields (Table1. entry 6). When lowering the temperature to $100^{\circ} \mathrm{C}$ we observed a significant decrease in product formation, and lowering the temperature further to $80{ }^{\circ} \mathrm{C}$ was associated with negligible product yield (Table1. entry 7 , 8). Finally, we tested the progress of reaction over time and found that the reaction exhibits similar conversions from two hours onward (Table1. entry 9).

With the optimized reaction conditions in hand, we sought to investigate the scope of reaction with respect to the ketone, aniline, and sulfonyl azide. First, different substituents on the aniline were examined revealing a broad scope of electron-donating or electron-withdrawing groups at the ortho, meta and para positions (Figure 1. examples 2a, 2e-2n). Aliphatic amines were also compatible with the reaction conditions (Figure 1. examples 20 and 2p). Next, we tested electron-donating and electron-withdrawing sulfonyl azides, which were tolerated under reaction conditions (Figure 1. examples $\mathbf{2 b - 2 d}$ ). The ketone scope was also broad, electron-withdrawing and electron-donating substituents provided good yields for meta and para substituted ketones, whereas substituents at the ortho position afforded diminished yields (Figure 1. examples 2q-2x). Finally, aliphatic, and benzylic ketones led to reasonable yields under our reaction conditions (Figure 1. examples $\mathbf{2 y}$ and $\mathbf{2 z}$ ). In the following section we discuss several of the trends that appear in the substrate scope. 

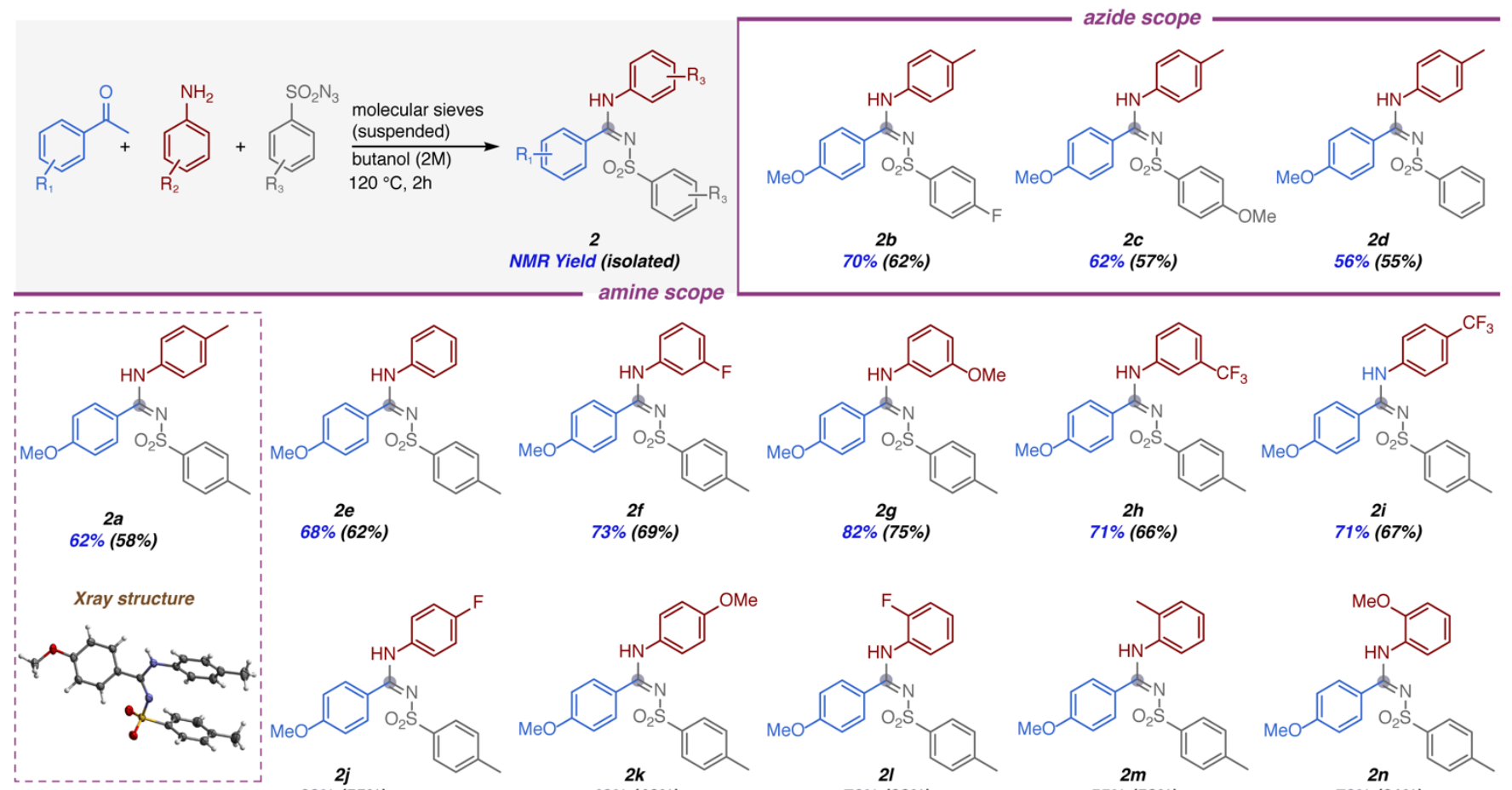
$2 e$
$68 \%(62 \%)$ $2 f$
$73 \%(69 \%)$ $2 g$
$82 \%(75 \%)$ $2 h$
$71 \%(66 \%)$

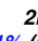
$2 i$
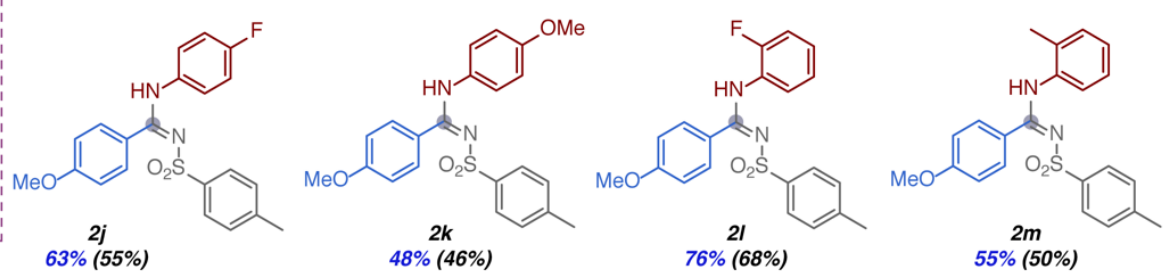

$55 \%(50 \%)$
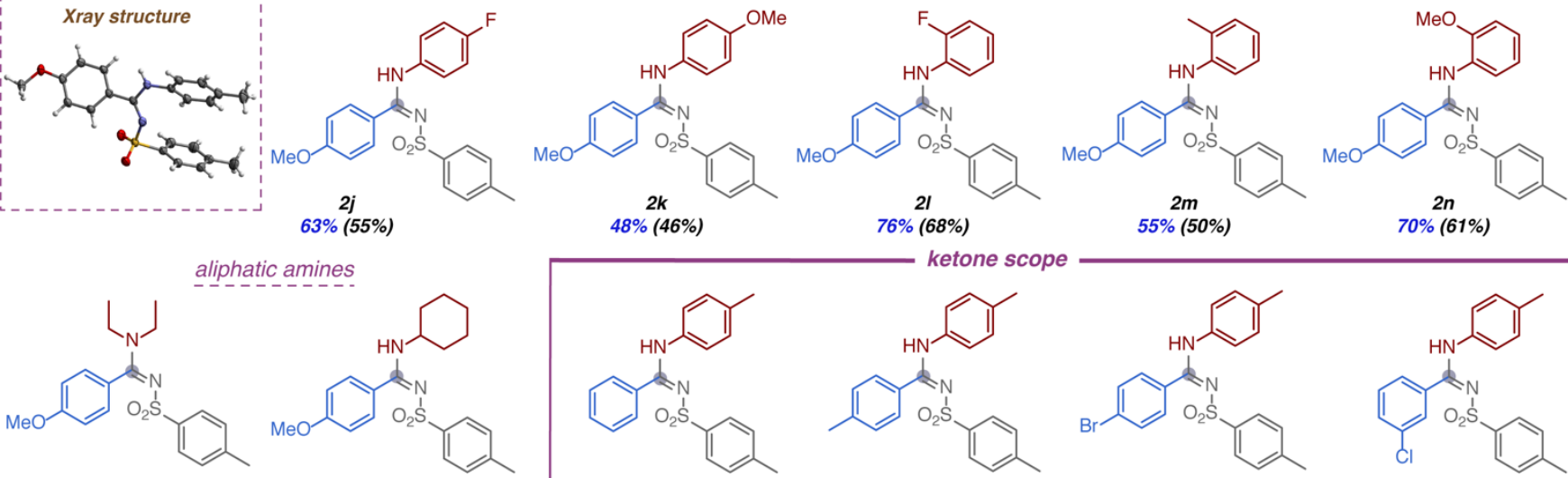

20
$54 \%(48 \%)$

$\underset{50 \%(41 \%)}{2 p}$

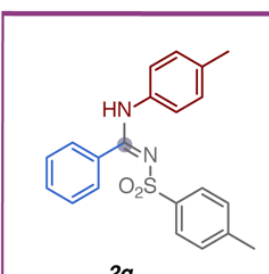

ketone scope

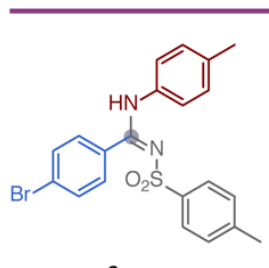

$70 \%(61 \%)$
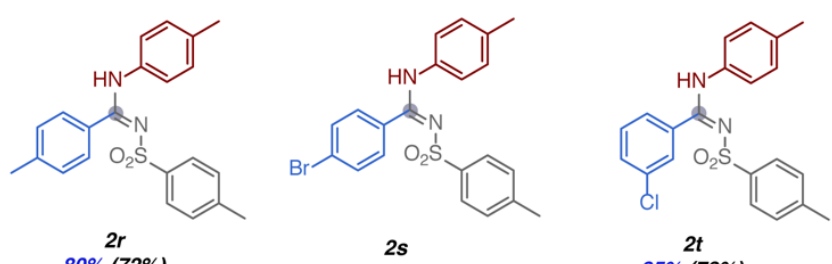
$\stackrel{2 q}{55 \%(48 \%)}$ $80 \%(72 \%)$ $\stackrel{2 s}{71 \%(65 \%)}$

benzylic and aliphatic ketones

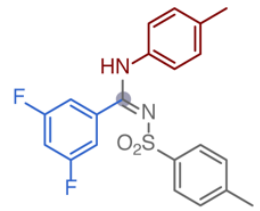

$2 u$
$9 \%(72 \%)$

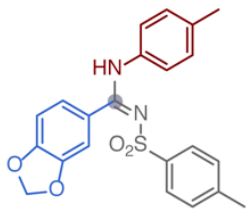

$2 v$

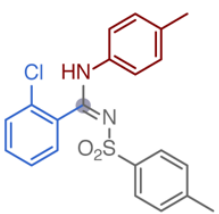

$2 w$
$25 \%(20 \%)$

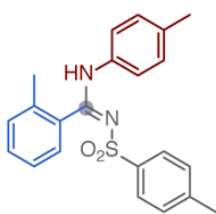

$2 x$
$25 \%(22 \%)$

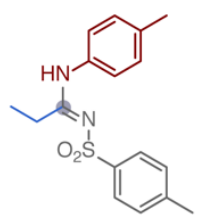

$2 y$
$54 \%(46 \%)$

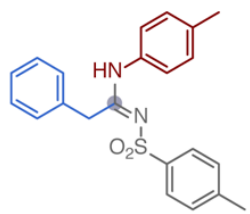

$2 z$ $56 \%(49 \%)$

Figure 1. scope of the amidine synthesis, reactions were conducted on a scale of $0.25 \mathrm{mmol}$ (ketone) and $0.35 \mathrm{mmol}$ (amine and azide). All reactions were performed in duplicate and the average yield is reported.

\section{Mechanism Evaluation}

According to the proposed reaction mechanism (see Figure 2.a.), imine (IM1) is first formed by a nucleophilic addition of an amine to the carbonyl group of a ketone under reaction conditions. ${ }^{24}$ The newly formed imine can then undergo an imine-enamine tautomerization to form an enamine intermediate (IM2). ${ }^{25,26}$ Sulfonyl azide reacts with the enamine via a dipolar cycloaddition to form a triazoline intermediate (IM3).12,27,28 Finally, the product is formed in a ring opening reaction together with the release of diazomethane gas. We first set out to verify the likelihood of the ring closing and opening steps by identifying the release of diazomethane. Based on literature precedents, ${ }^{28,29}$ we aimed to intercept diazomethane in situ by reacting it with benzoic acid, which was added to the reaction mixture to afford the corresponding ester (See Figure 2.b. and Scheme S4 in SI for further details). Methyl benzoate was indeed detected under these modified reaction conditions and amidine formation was not affected by the presence of benzoic acid leading to similar yields, which indicated that the cyclization/de-cyclization steps in the mechanism were plausible.

Examination of the reaction scope reveals that structural features of the aniline component greatly impact the reaction yield. Thus, we wished to identify which of the reaction steps is influenced by the aniline substitution pattern. The role of aniline in the mechanism is unique, it first behaves as a nucleophile that adds to ketone to form imine; thus, anilines bearing electron-donating groups should increase reactivity in this step. However, electron-donating aniline-derived intermediates would not facilitate the following tautomerization and cycloaddition steps. Based on this dichotomy, aniline served as an ideal probe for exploring the proposed reaction mechanism and identifying which steps are associated with reactivity. We first attempted to correlate Hammett parameters to the yield of reaction using meta- and para-substituted anilines. ${ }^{30-32}$ 
a. Proposed mechanism

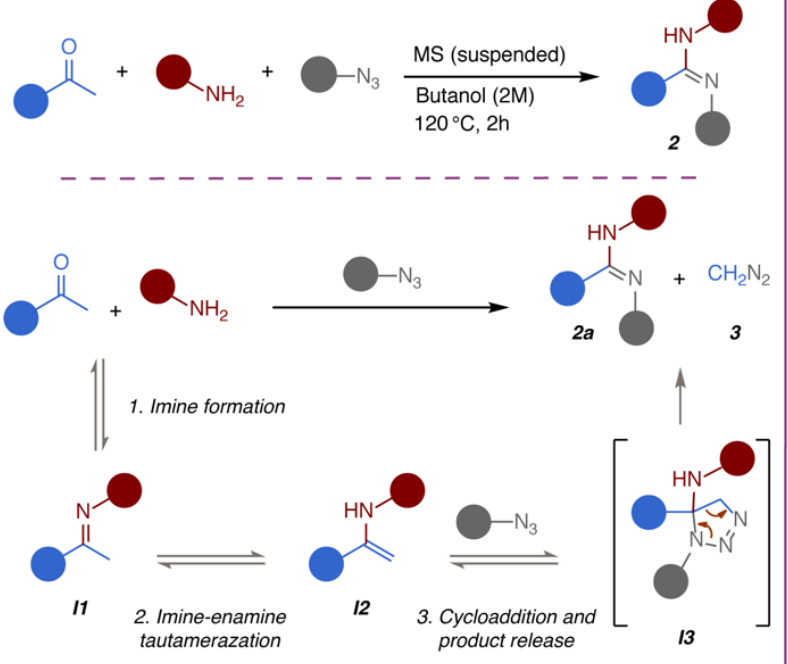

b. __ Trapping the diazomethane byproduct

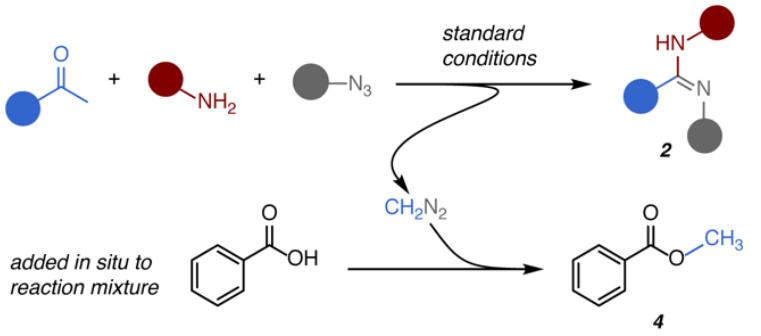

g. Substituent effect on the distal aceto-position

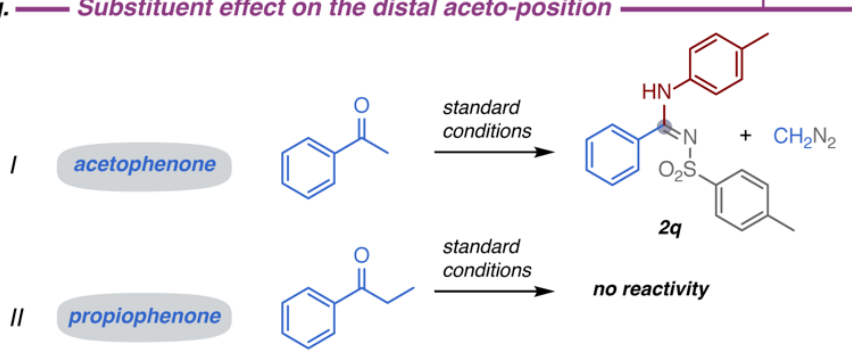

propiopher

i. - Hypothesized challenges associated with propiophenone reactivity
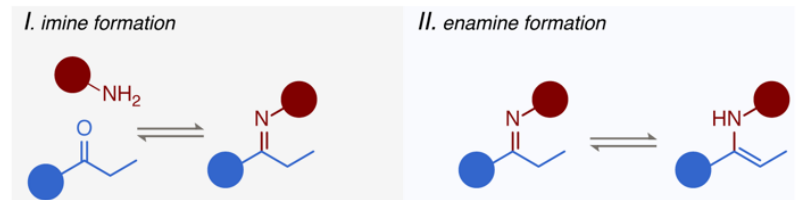

III. cycloaddition

IIII. product release
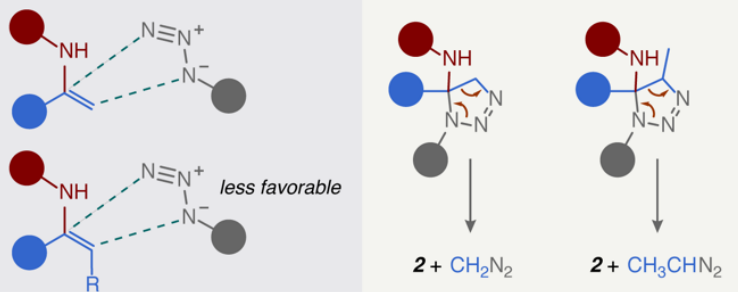

Figure 2. (a) General reaction scheme and proposed mechanism effect of different anilines on the yield (d) Measured versus predicted yield values, the goodness-of-fit is represented by $\mathrm{R}^{2}$ and $\mathrm{Q}^{2}$ for leave one-out and 3-fold validation (see SI section 6 for details) (e) Model descriptors: $v$, ring vibration, and $\mu$, NPA charge on the carbon adjacent to the amine group (f) Representation of the iminium intermediate (g) The difference between acetophenone and propiophenone in reactivity $(\mathrm{h})$ Investigating imine formation under standard reaction conditions (i) Mechanistic hypotheses for the lack of propiophenone reactivity (j) Investigating imine-enamine tautomerization and cycloaddition steps with acetophenoneand propiophenone-derived intermediates

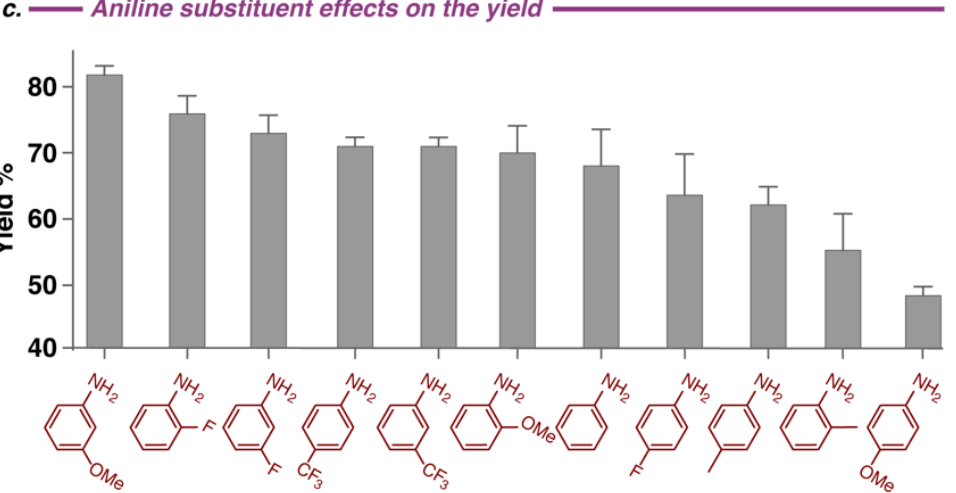

d. Plausible transition state $\square$ f. - Multivariate statistical model

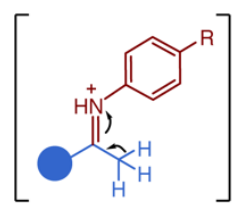

Iminium intermediate

- Predictive parameters
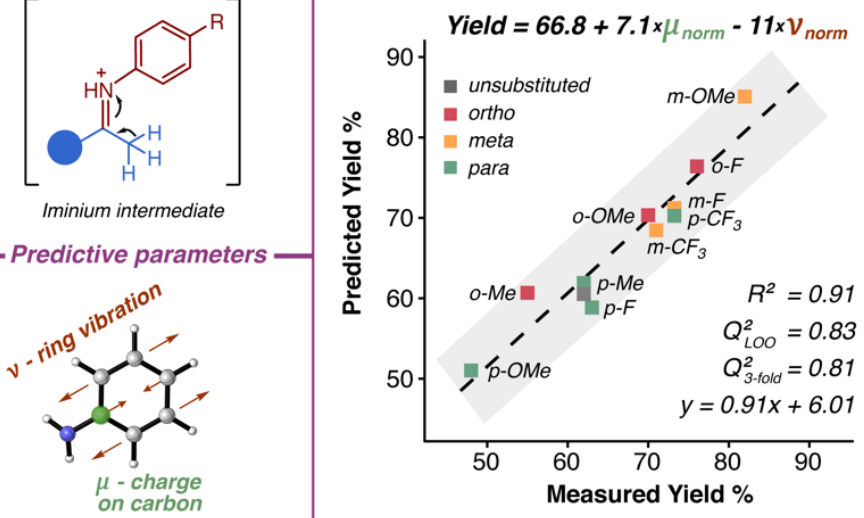

h. Imine formation under reaction conditions

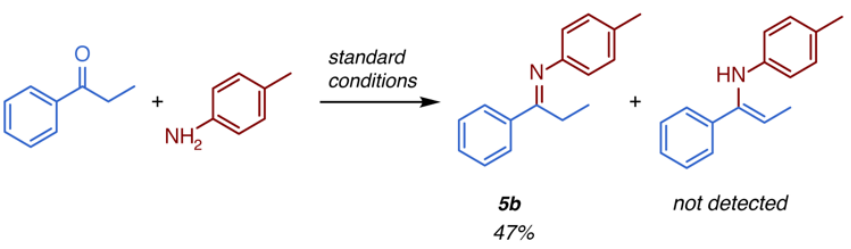

j. Enamine formation and dipolar-cycloaddition

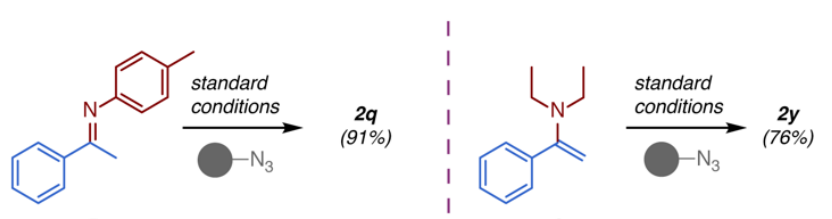

$5 a$

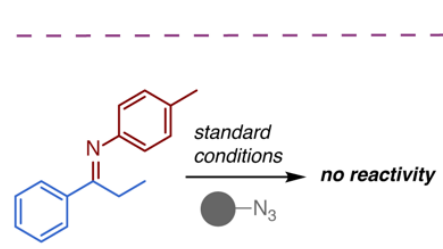

$5 b$

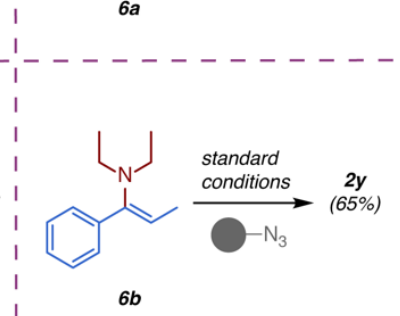


In the absence of the $m$-anisidine, we identified a good correlation with $\sigma+$ values (see SI Figure S5 for details). The slope of the plot was positive, which classically indicates the formation of a negative charge or the collapse of a positive charge at the rate-determining step. This analysis fits well with our observation that electron-withdrawing substituents increase reactivity given a collapse of positive charge at the postulated iminium transition state (Figure 2.d.). ${ }^{33}$ Thus, this analysis supports the formation of enamine as being rate-limiting. Moreover, the poor fit with $\sigma$ values and the reduced correlation with $m$-anisidine included, both support the important contribution of resonance to the rate-determining transition state(s).

Given these limitations of the Hammett correlation and the fact that we also had ortho-substituted anilines in our set, we selected to apply a multivariate approach. ${ }^{34,35}$ A robust model with two parameters was identified: the natural population analysis (NPA) charge on the ring carbon connected to the nitrogen and a ring vibration aligned with the para substituent axis ( $\mu$ and $v$ respectively, Figure 2.e.). ${ }^{36}$ In this model increased positive charge adjacent to the nitrogen and lower vibration frequencies lead to higher yields. The vibration frequencies are difficult to interpret intuitively because they are influenced by changes to charge and the mass and location of the substituents (see SI Figure S8 for further details). However, the variation in charge confirms the conclusions gained from the Hammett analysisthe ability of substituents to stabilize the iminium transition state, through both resonance and inductive effects, is responsible for the yield of the reaction (See Figure 2.d-f.).

\section{Regioselectivity}

Prior to intercepting the diazomethane in situ, we speculated that by replacing the methyl group of acetophenone with extended alkyl substituents, we would be able to isolate non-gaseous diazoalkane by-products. Interestingly, upon the addition of propiophenone to the reaction mixture instead of acetophenone (See Figure 2.g.), product was not formed and the ketone remained unreacted. This result does not represent a limitation of our methodology because both substrates lead to the same product with a different by-product. Yet, the origin of this puzzling observation might shed light on the reaction mechanism. We postulated that the lack of propionate reactivity could be associated with one of the following challenges (Figure 2.i.): (a) imine formation might be prohibitive, (b) the tautomerization step to form enamine may be unfavorable, (c) bulkier substituents on the enamine might limit the approach of sulfonyl azides, and (d) the release of bulkier diazoalkanes may be less favorable than the release of diazomethane gas.

To differentiate between these mechanistically distinct options, we tried to intercept the proposed intermediates and submit each of them to our reaction conditions. We first studied the formation of imine and enamine when treating propiophenone with aniline in the absence of azide. Under our reaction conditions, imine was observed while enamine was not detected (Figure 2.h.). We then probed the imineenamine tautomerization step by synthesizing and purifying the imines of acetophenone and propiophenone, followed by treatment with azide under our standard reaction conditions (Figure 2.j.). Product formation was observed with acetophenone, whereas the propiophenone imine remained unreacted. We then sought to test whether the reaction can proceed upon the addition of azide to enamines. The enamines that were postulated to form under our reaction conditions are not stable, thus we synthesized and isolated stable enamines from secondary amines with acetophenone (6a) and propiophenone (6b) and submitted them to our reaction conditions (Figure 2.j.). ${ }^{37,38}$ In both cases, amidine products were obtained, implying that the cyclization/de-cyclization steps are not limiting to propiophenone reactivity. Overall, this set of experiments strongly suggested that whereas propiophenone can form an imine, it could not proceed to form enamine under our reaction conditions.

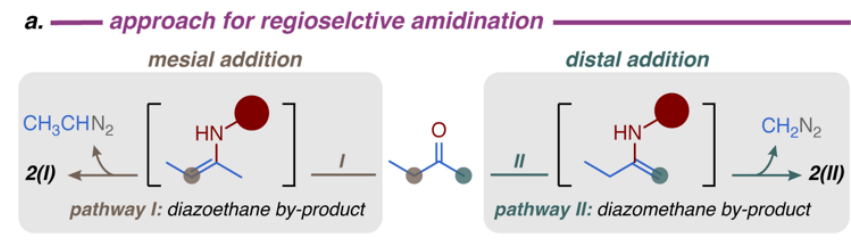

b. - regioselective amidination of aliphatic and benzylic substrates
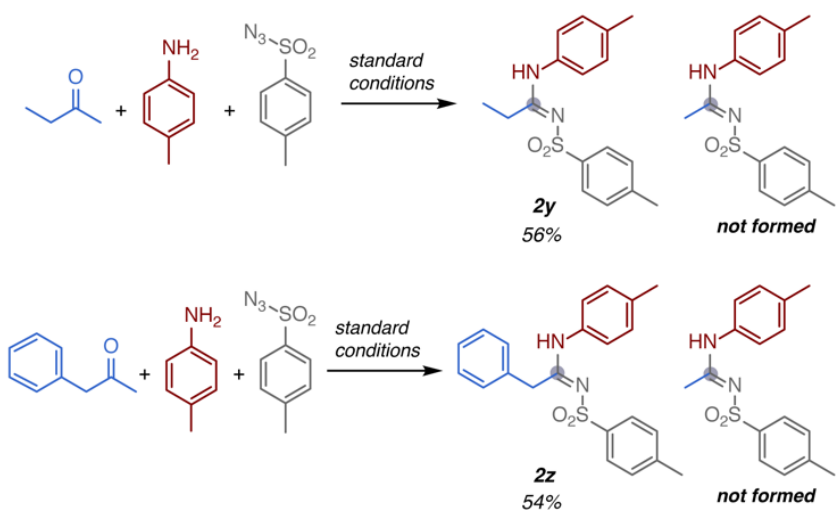

Figure 3. (a) General approach for regioselective amidination (b) Proof-of-concept: aliphatic and benzylic substrates demonstrate regioselectivity

Given these results, we postulated that the sensitivity of the reaction to the nature of the alkyl substituent on the ketone could provide us with a powerful methodology to control regioselective amidine transformations of aliphatic substrates. Upon the addition of aliphatic ketones, theoretically, the reaction may proceed through two possible pathways (see figure 3.a.): (I) the imine-enamine tautomerization would occur on the mesial position with the elimination of the corresponding diazoalkane by-product; (II) the imine-enamine tautomerization would occur on the distal methyl position with the elimination of diazomethane. As a proof-of-concept we submitted butanone to our reaction conditions to probe its regioselectivity. Indeed, the tautomerization occurs only on the distal methyl position, leaving the mesial position intact and providing a highly regioselective transformation (2y Figure3.b.). Similar behavior was observed with phenyl acetone as substrate affording product 2z exclusively (Figure3.b.).

\section{Applications and Conclusion}

To demonstrate the applicability of our methodology, we scaled up the reaction using $\mathbf{1 a}, \mathbf{1 d}$, and $\mathbf{1 c}$ as model substrates. With industrial applications in mind, we selected to run this reaction under solvent-free conditions affording $67 \%$ isolated yield (Figure 4.a.). Moreover, as the costs of 
large scale columns are prohibitive, ${ }^{39}$ we opted to recrystallize the product, which is facilitated by the solvent-free conditions in this reaction set-up. Next, we aimed to apply this methodology in late-stage functionalization to create a specific structural core found in a wide variety of biological active molecules. Namely, we constructed in a single step the amidine core of a glibenclamide-derivative, which is a very commonly prescribed diabetes medication. ${ }^{40-42}$ The ketone and amine components were commercially available, and we synthesized the required sulfonyl azide in three steps (See SI section 3.8 for details). We then submitted the three components to our standard reaction conditions to afford the desired product 7 in $52 \%$ isolated yield (Figure 4.b.). ${ }^{43}$
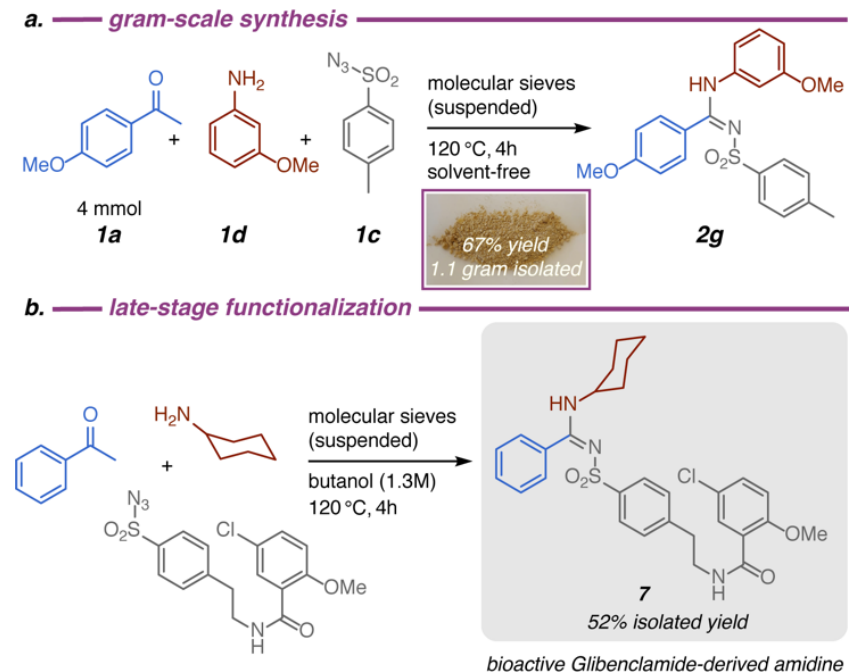

Figure 4. (a) Gram-scale synthesis of $\mathbf{2 g}$ under solvent-free conditions (b) Glibenclamide-derivative synthesis by coupling the three components in a single step

In summary, we have developed a novel methodology for the synthesis of sulfonyl amidines from readily available starting materials in a metal-free one-pot reaction. These reaction conditions afford a broad substrate scope and functional group tolerance. Studies focused on intercepting reaction intermediates and submitting them to our reaction conditions provide insights on the reaction mechanism, which are further supported by Hammett analysis and multivariate linear regression models. Moreover, this mechanistic analysis led to the discovery of a highly regioselective amidination of aliphatic ketones. The one-step, multicomponent synthesis of a bioactive glibenclamide derivative demonstrated the efficacy of this methodology in drug development.

\section{ASSOCIATED CONTENT}

Supporting Information. Electronic Supplementary Information (ESI) available free of charge via the Internet at http://pubs.acs.org. All underlying data are available in the article itself and its supplementary materials and include: Detailed experimental and computational procedures, analyses, and characterizations (AmidineSI.pdf); Excel sheet with the results and parameters that were used to identify models (Amidine2022.xlsx); Zip archive with cif and checkcif X-ray files (xray.zip). The raw data and code files are openly available in GitHub at https://github.com/Milo-group/Amidine2022 and include machine readable NMR and HRMS traces, as well as all optimized .xyz files, code and data sets.

\section{AUTHOR INFORMATION}

\section{Corresponding Author}

*correspondence should be addressed to anatmilo@bgu.ac.il.

\section{Author Contributions}

The manuscript was written through contributions of all authors, who have given approval to the final version of the manuscript.

\$These authors contributed equally.

\section{Funding Sources}

This research was supported by the Israel Science Foundation (Grants No. 2252/21).

\section{Notes}

The authors declare no competing financial interest.

\section{ACKNOWLEDGMENT}

Z.A. acknowledges the Kreitman Graduate School for the chemo-tech scholarship and the Council for Higher Education for the PBC scholarship. A.A.R. acknowledges the Kreitman Graduate School for the chemo-tech scholarship. The authors thank Vasudevan Dhayalan for his assistance with preliminary experiments; Radion Vainer for X-ray analysis; Shahar Barkai for parameter extraction and model development codes; and Doron Pappo for fruitful discussions.

\section{REFERENCES}

(1) Galloway, W. R. J. D.; Isidro-Llobet, A.; Spring, D. R. DiversityOriented Synthesis as a Tool for the Discovery of Novel Biologically Active Small Molecules. Nat. Commun. 2010, 1 (6), 113.

(2) Schreiber, S. L. Target-Oriented and Diversity-Oriented Organic Synthesis in Drug Discovery. Science (80-. ). 2000, 287 (5460), 1964-1969.

(3) Galloway, W. R. J. D.; Spandl, R. J.; Bender, A.; Thomas, G. L.; DiazGavilan, M.; O'connell, K. M. G.; Spring, D. R. Diversity-Oriented Synthesis. Chem. Genomics 2012, 39-59.

(4) Burke, M. D.; Schreiber, S. L. A Planning Strategy for DiversityOriented Synthesis. Angew. Chemie - Int. Ed. 2004, 43 (1), 46-58.

(5) Armstrong, R. W.; Combs, A. P.; Tempest, P. A.; Brown, S. D.; Keating, T. A. Multiple-Component Condensation Strategies for Combinatorial Library Synthesis. Acc. Chem. Res. 1996, 29 (3), 123-131.

(6) Slobbe, P.; Ruijter, E.; Orru, R. V. A. Recent Applications of Multicomponent Reactions in Medicinal Chemistry. Medchemcomm 2012, 3 (10), 1189-1218.

(7) Tietze, L. F.; Modi, A. Multicomponent Domino Reactions for the Synthesis of Biologically Active Natural Products and Drugs. Med Res. Rev. 2000, 20 (4), 304-322.

(8) Wang, M. J.; Liu, Y. Q.; Chang, L. C.; Wang, C. Y.; Zhao, Y. L.; Zhao, X. B.; Qian, K.; Nan, X.; Yang, L.; Yang, X. M.; Hung, H. Y.; Yang, J. S.; Kuo, D. H.; Goto, M.; Morris-Natschke, S. L.; Pan, S. L.; Teng, C. M.; Kuo, S. C.; Wu, T. S.; Wu, Y. C.; Lee, K. H. Design, Synthesis, Mechanisms of Action, and Toxicity of Novel 20(s)Sulfonylamidine Derivatives of Camptothecin as Potent Antitumor Agents. J. Med. Chem. 2014, 57 (14), 6008-6018.

(9) Quek, J. Y.; Davis, T. P.; Lowe, A. B. Amidine Functionality as a Stimulus-Responsive Building Block. Chem. Soc. Rev. 2013, 42 (17), 7326-7334.

(10) Greenhill, J. V; Lue, P. Amidines and Guanidines in Medicinal Chemistry. Prog. Med. Chem. 1993, 30, 203-326.

(11) Patai Rappoport, Zvi., S. The Chemistry of Amidines and Imidates. Wiley. 1991.

(12) Citerio Saccarello, Maria Luisa, Stradi, Riccardo, L. Direct Synthesis of 3-Morpholino-2,3-Dihydroindoles from Enamines and Aryl Azides. 2002.

(13) Sollenberger, P. Y.; Martin, R. B. Mechanism of Enamine 
Hydrolysis. J. Am. Chem. Soc. 1970, 92 (14), 4261-4270.

(14) Croce, P. D.; Stradi, R. V-Triazolines-VI11Part V. D. Pocar and P. Trimarco, J. Chem. Soc. Perkin I, 622 (1976).: Rearrangement of Triazolines from 2-Substituted-1-Aryl-and 1-Heteroaryl-1Amino-Ethylenes and Tosylazide. Tetrahedron 1977, 33 (8), 865867.

(15) Breunig, H. J.; Burford, N.; Rösler, R. A Palladium-Catalyzed Synthesis of Amidines from Aryl Halides. Angew. Chemie - Int. Ed. 2000, 39 (22), 4156-4158.

(16) Bae, I.; Han, H.; Chang, S. Highly Efficient One-Pot Synthesis of NSulfonylamidines by $\mathrm{Cu}$-Catalyzed Three-Component Coupling of Sulfonyl Azide, Alkyne, and Amine. J. Am. Chem. Soc. 2005, 127 (7), 2038-2039.

(17) Zhang, Z.; Huang, B.; Qiao, G.; Zhu, L.; Xiao, F.; Chen, F.; Fu, B.; Zhang, Z. Tandem Coupling of Azide with Isonitrile and Boronic Acid: Facile Access to Functionalized Amidines. Angew. Chemie Int. Ed. 2017, 56 (15), 4320-4323.

(18) Lange, U. E. W.; Schäfer, B.; Baucke, D.; Buschmann, E.; Mack, H. A New Mild Method for the Synthesis of Amidines. Tetrahedron Lett. 1999, 40 (39), 7067-7070.

(19) Takuwa, T.; Minowa, T.; Onishi, J. Y.; Mukaiyama, T. Facile One-Pot Syntheses of Amidines and Enamines from Oximes via Beckmann Rearrangement Using Trifluoromethanesulfonic Anhydride. Bull. Chem. Soc. Jpn. 2004, 77 (9), 1717-1725.

(20) Croce Stradi, Riccardo, P. D. V-TriazolinesVI 11 Part V. D. Pocar and P. Trimarco, J. Chem. Soc. Perkin I, 622 (1976). :Rearrangement of Triazolines from 2-Substituted-1-Aryland 1-Heteroaryl-1-Amino-Ethylenes and Tosylazide. Tetrahedron 1977, 33 (8), 865-867.

(21) Zuckerman, R. L.; Krska, S. W.; Bergman, R. G. Zirconium-Mediated Metathesis of Imines: A Study of the Scope, Longevity, and Mechanism of a Complicated Catalytic System. J. Am. Chem. Soc. 2000, 122 (5), 751-761.

(22) Reddelien, G. Zinc Chloride as a Condensing Agent. Justus Liebig's Annalen der Chemie und Pharmacie. CF Winter'sche,: Leipzig : pp 165-199, 1912

(23) Schiff, H. Eine Reihe. European J. Org. Chem. 1864, 131, 118-119.

(24) Rabjohn , N. Organic Syntheses. Collective Volume 4 Collective Volume 4; Wiley: New York, 1990.

(25) Wei, Y.; Deb, I.; Yoshikai, N. Palladium-Catalyzed Aerobic Oxidative Cyclization of N-Aryl Imines: Indole Synthesis from Anilines and Ketones. J. Am. Chem. Soc. 2012, 134 (22), 90989101.

(26) Babler, J. H.; Atwood, M. C.; Freaney, J. E.; Viszlay, A. R. The Role of Imine-Enamine Tautomerism in Effecting Cross-Aldol Condensations. Tetrahedron Lett. 2007, 48 (43), 7665-7667.

(27) Delanty, G. The Chemistry. Lit. Imagin. 2010, 12 (3), 289-290.

(28) Chang, S.; Lee, M.; Jung, D. Y.; Yoo, E. J.; Cho, S. H.; Han, S. K. Catalytic One-Pot Synthesis of Cyclic Amidines by Virtue of Tandem Reactions Involving Intramolecular Hydroamination under Mild Conditions. J. Am. Chem. Soc. 2006, 128 (38), 12366-12367.

(29) Xu, X.; Ge, Z.; Cheng, D.; Lei, M.; Lu, C.; Zhang, Q.; Yao, N.; Li, X.
CuCI/CCI4-Promoted Convenient Synthesis of Sulfonyl Amidines from Tertiary Amines and Sulfonyl Azides. Org. Lett. 2010, 12 (5), 897-899.

(30) Hammett, L. P. The Effect of Structure upon the Reactions of Organic Compounds. Temperature and Solvent Influences.J. Chem. Phys. 1936, 4 (9), 613-617.

(31) Hansch, C.; Leo, A.; Taft, R. W. A Survey of Hammett Substituent Constants and Resonance and Field Parameters. Chem. Rev. 1991 91 (2), 165-195.

(32) Jacobsen, E. N.; Zhang, W.; Guler, M. L. Electronic Tuning of Asymmetric Catalysts. J. Am. Chem. Soc. 1991, 113 (17), 67036704.

(33) Anslyn Dougherty, Dennis A., E. V. Modern Physical Organic Chemistry; University Science: Sausalito, California, 2006.

(34) Dhayalan, V.; Gadekar, S. C.; Alassad, Z.; Milo, A. Unravelling Mechanistic Features of Organocatalysis with in Situ Modifications at the Secondary Sphere. Nat. Chem. 2019, 11 (6), 543-551.

(35) Milo, A.; Bess, E. N.; Sigman, M. S. Interrogating Selectivity in Catalysis Using Molecular Vibrations. Nature 2014, 507 (7491) 210-214.

(36) Glendening, E. D.; Badenhoop, J. K.; Reed, A. E.; Carpenter, J. E.; Bohmann, J. A.; Morales, C. M.; Karafiloglou, P.; Landis, C. R. Weinhold, F. NBO7. Theoretical Chemistry Institute, University of Wisconsin Madison, WI, USA 2018.

(37) Hattori, G.; Yoshida, A.; Miyake, Y.; Nishibayashi, Y. Enantioselective Ring-Opening Reactions of Racemic Ethynyl Epoxides via Copper-Allenylidene Intermediates: Efficient Approach to Chiral $\beta$-Amino Alcohols. J. Org. Chem. 2009, 74 (20), 7603-7607.

(38) Gao, T.; Zhao, M.; Meng, X.; Li, C.; Chen, B. Facile Synthesis of Sulfonyl Amidines and $\beta$-Amino Sulfonyl Enamines under Transition-Metal-Free Conditions. Synlett 2011, No. 9, 12811284.

(39) Antenucci, A.; Dughera, S.; Renzi, P. Green Chemistry Meets Asymmetric Organocatalysis: A Critical Overview on Catalysts Synthesis. ChemSusChem 2021, 14 (14), 2785-2853.

(40) Simard, J. M.; Chen, M.; Tarasov, K. V.; Bhatta, S.; Ivanova, S.; Melnitchenko, L.; Tsymbalyuk, N.; West, G. A.; Gerzanich, V. Newly Expressed SUR1-Regulated NCCa-ATP Channel Mediates Cerebral Edema after Ischemic Stroke. Nat. Med. 2006, 12 (4), 433-440.

(41) England, T. N. Numb Er 26. 2000, 347 (20), 1549-1556.

(42) Kahn, S. E.; Haffner, S. M.; Heise, M. A.; Herman, W. H.; Holman, R. R.; Jones, N. P.; Kravitz, B. G.; Lachin, J. M.; O’Neill, M. C.; Zinman B.; Viberti, G. Glycemic Durability of Rosiglitazone, Metformin, or Glyburide Monotherapy. N. Engl. J. Med. 2006, 355 (23), 24272443.

(43) This is the best yield to date compared to the $42 \%$ obtained with rhodium catalysis in reference 17 . 


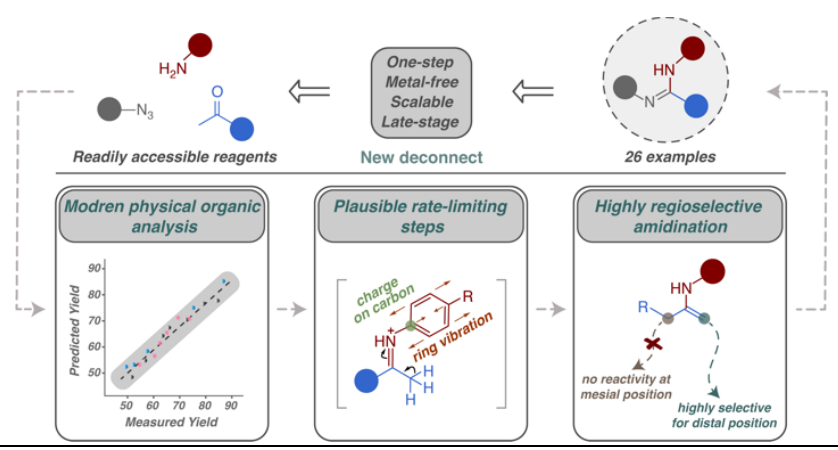

